\title{
Effect of experimental design parameters on mechanical properties of braided stentriever made from shape memory alloy
}

\author{
Narongdet Sulatchaneenopdon ${ }^{1, a^{*}}$, Anak Khantachawana ${ }^{1,2, b}$ \\ ${ }^{1}$ Department of Mechanical Engineering, Faculty of Engineering, \\ ${ }^{2}$ Biological Engineering Program, Faculty of Engineering, \\ King Mongkut's University of Technology Thonburi, Thailand \\ 126 Pracha Uthit Rd., Bang Mod, Thung Khru, Bangkok 10140, Thailand \\ anarongdet.sulat@gmail.com, banak.kha@kmutt.ac.th
}

\begin{abstract}
Keywords: Self-expandable stents, Braiding technology, Mechanical properties, Radial resistive force, Shape memory
\end{abstract}

\begin{abstract}
This research aims to study the effects of experimental design parameters such as the number of wires, the length and braiding angle of stent on mechanical properties of braiding stentriever made from shape memory alloy. Self-expanding stents or stentriever were fabricated by braiding a number of wires together by braiding machine, followed by heat-treatment for shape setting. Superelastic NiTi shape memory alloy wires with diameters of $0.08 \mathrm{~mm}$ were used while the number of wires was selected to be 12, 24 and 36, respectively. Braiding angle was designed to be $52 \mathrm{o} \pm 2 \mathrm{o}$ and $38 \mathrm{o} \pm 2 \mathrm{o}$. Diameter of stentriever was set to be $4 \mathrm{~mm}$. On the other hand, this design is focused on stent using in $3 \mathrm{~mm}$ to $4 \mathrm{~mm}$ intracranial vessel diameter stent, radial resistive test, radial expansion test and three-point bending test under temperature at $37 \mathrm{o}$ are carried out. The result shows that the maximum radial resistive force, maximum radial expansion force and maximum bending force which are equaled to $50 \mathrm{mN} / \mathrm{mm}, 37 \mathrm{mN} / \mathrm{mm}$ and $0.423 \mathrm{~N}$, respectively can be confirmed in stent with 36 wires.
\end{abstract}

\section{Introduction}

At present the cerebrovascular disease or stroke is the third most common causes of death of the population around the world. It is estimated that every 6 seconds someone dies from a stroke or 6 million in 15 million peoples' deaths from a stroke since 2013 [1]. The acute management of ischemic stroke is restoring blood flow to the affected area as quickly as possible to reduce longterm disability [2]. Stentriever were mainly used as the most effective devices for intracranial thrombectomy [2]. A conventional retriever stent is produced from Nickel-Titanium base shape memory alloy by laser-cutting technology from tube or plate as a raw material. However, lasercutting process has disadvantage since the cost is expensive and heat effect from laser can change properties of shape memory behavior which is very sensitive to heat-treatment [3-4]. Therefore, braiding process has benefited when manufacturing in large scale and easy to control the property of stent itself.

The aim of this research is to investigate the effects of experimental design parameters in braiding process of such as number of wires, braiding angle on mechanical properties of the prototype stentriever made from NiTi shape memory alloys. Moreover, Radial resistive force and radial expansion force and three-point bending force were measured in order to evaluate the mechanical properties of the stent according to ASTM standard.

\section{Materials and Method}

The commercial Ti-50.8 Ni at\% shape memory alloy wires with diameters of $0.08 \mathrm{~mm}$ were prepared in order to used as raw material. Stents were fabricated by braiding on metal rod with braiding machine. Shape setting was conducted by heat-treatment at $803 \mathrm{~K}$ for $10 \mathrm{~min}$. Phase transformation temperatures were investigated by differential scanning calorimeter (DSC) under 
temperature range between -50 to $100{ }^{\circ} \mathrm{C}$ with cooling and heating rate of $10{ }^{\circ} \mathrm{C} / \mathrm{min}$. The stent was fabricated by braiding machine and the number of wire can be controlled by changing the number of monofilament $(12,24$ and 36 wire) while braiding angle can be controlled by changing the taking up speed of braiding machine $\left(52^{\circ} \pm 2^{\circ}\right.$ and $\left.38^{\circ} \pm 2^{\circ}\right)$. The physical characteristic of stent such as braiding pattern was observed using optical microscope (Olympus NX-5100) as shown in Fig. 1. The radial resistive force and radial expansion force were determined under ASTM F-3067. The stent was loaded to reduce diameter to $25 \%$ of nominal diameter and the loading and unloading force were measured. The flexibility behavior was determined by three-point bending test which was performed under ASTM F2507. The maximum load at maximum deflection of $3.2 \mathrm{~mm}$ was used to evaluate. All the mechanical tests were carried out with micro tensile machine MultiTest-x.
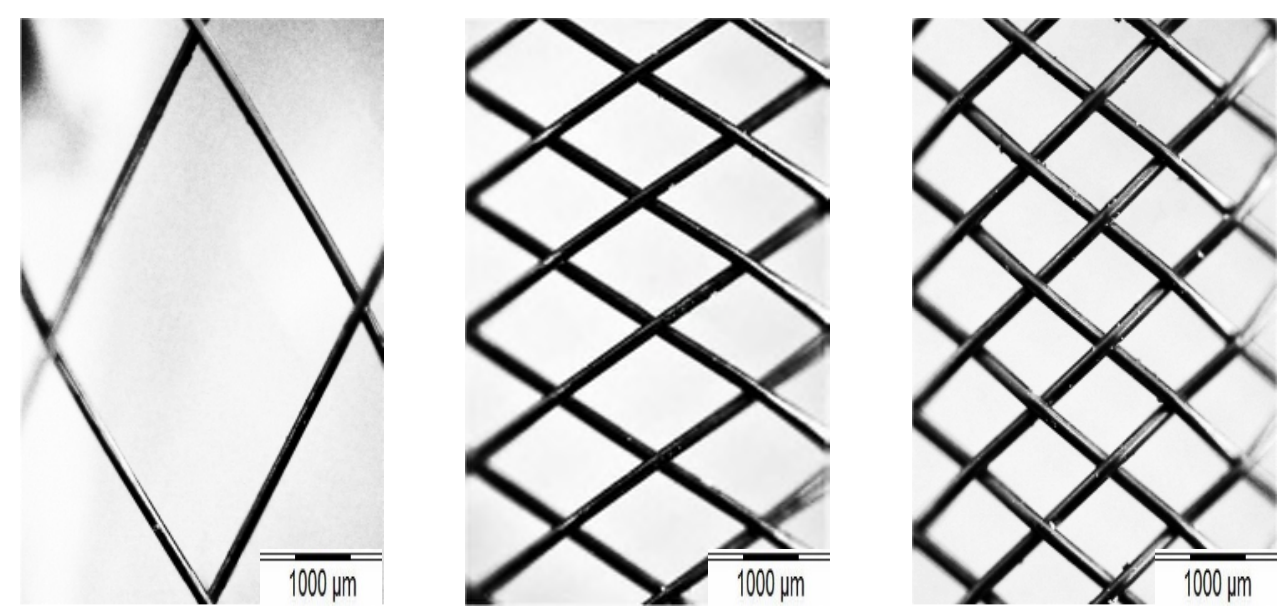

Fig. 1 Stent fabricated with various wire number of 12, 24 and 36.

\section{Results and Discussions}

Transformation Temperature. Transformation temperatures of fabricated NiTi stent were detected by Differential scanning calorimetry (DSC). In order to obtain superelasticity at body temperature, transformation temperatures are required to be lower than body temperature. The heatflow under cooling and heating obtained from NiTi wire heat treated at $803 \mathrm{~K}$ for 10 mins shows that Af temperature is $18{ }^{\circ} \mathrm{C}$ which is below $37^{\circ} \mathrm{C}$. Moreover, temperature hysteresis is $5^{\circ} \mathrm{C}$ which is relatively small, though the DSC curves are not shown here.

Braiding Angle. Braiding angle was observed by an optical microscope. Fig. 1 shows microimages of stent fabricated with various number of wires after shape setting process. It is noted that the braiding angles can be varied by changing take up speed from take up motor. The braiding angles of $52^{\circ} \pm 2^{\circ}$ and $38^{\circ} \pm 2^{\circ}$ were investigated at intersection of wire in vertical directions and shown in Fig. 2. It is also noted that the structure of the stent can be constructed only when the take up speed is lower than $85.6 \mathrm{~mm} / \mathrm{s}$.
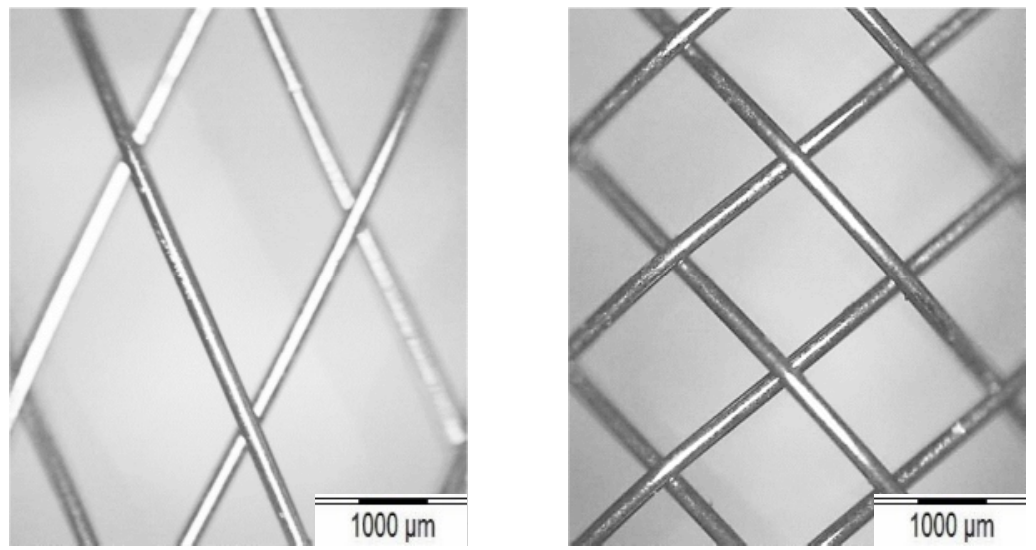

Fig. 224 wire-stent with braiding angle of $38^{\circ}$ and $52^{\circ}$. 


\section{Bending Behavior}

The load-deflection curves of stent is used to identify the bending characteristics of the stent during movement in the torturous blood vessel. Fig. 3 shows the bending behavior of braiding stentriever with various number of wires and braiding angle. It is obvious that with increasing the braiding angle and number of wires resulting in higher bending force. On the other hand, it seems that number of wires have shown a greater difference in bending force than braiding angle. It is clear from Fig. 4 that stent with 36 wires reveals the maximum bending force in vessel with radius of curvature of $3.2 \mathrm{~mm}$.

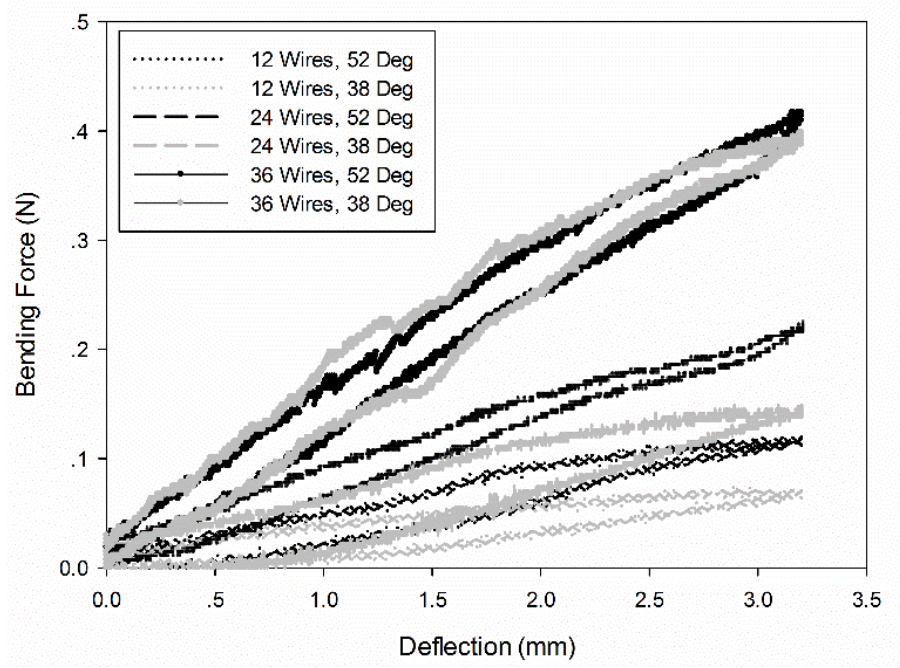

Fig. 3 Load-deflection curves of stents fabricated with various number of wires and different braiding angles.

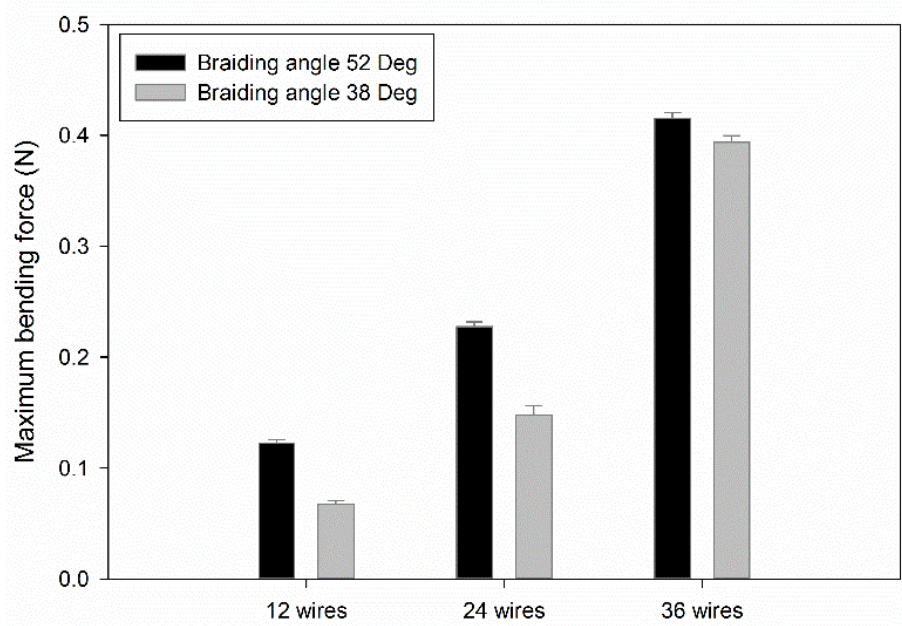

Fig. 4 maximum bending force at $3.2 \mathrm{~mm}$ deflection obtained from stentriever with various number of wires and braiding angles.

\section{Radial Behavior}

Fig. 5 shows the radial behavior of stent with various number of wires and braiding angle obtained during circumferential compression load and expansion load. This behavior is very important for evaluate the performance of stent under compression force and expansion force from movement of blood vessel after delivery. Fig. 6 shows those values of 12, 24 and 36 wired-stent, respectively. It is seen that with increasing braiding angle and the number of wires, the average radial resistive force and average radial expansion increase. It is seen that effect of the number of wires on resistive force seems to be greater than those of braiding angleThe highest value of radial 
resistive force and radial expansion force were $49.57 \mathrm{~N}$ and $36.83 \mathrm{~N}$, respectively obtained from 36 wire stent with a braiding angle of 52 degree as shown in Fig. 6.

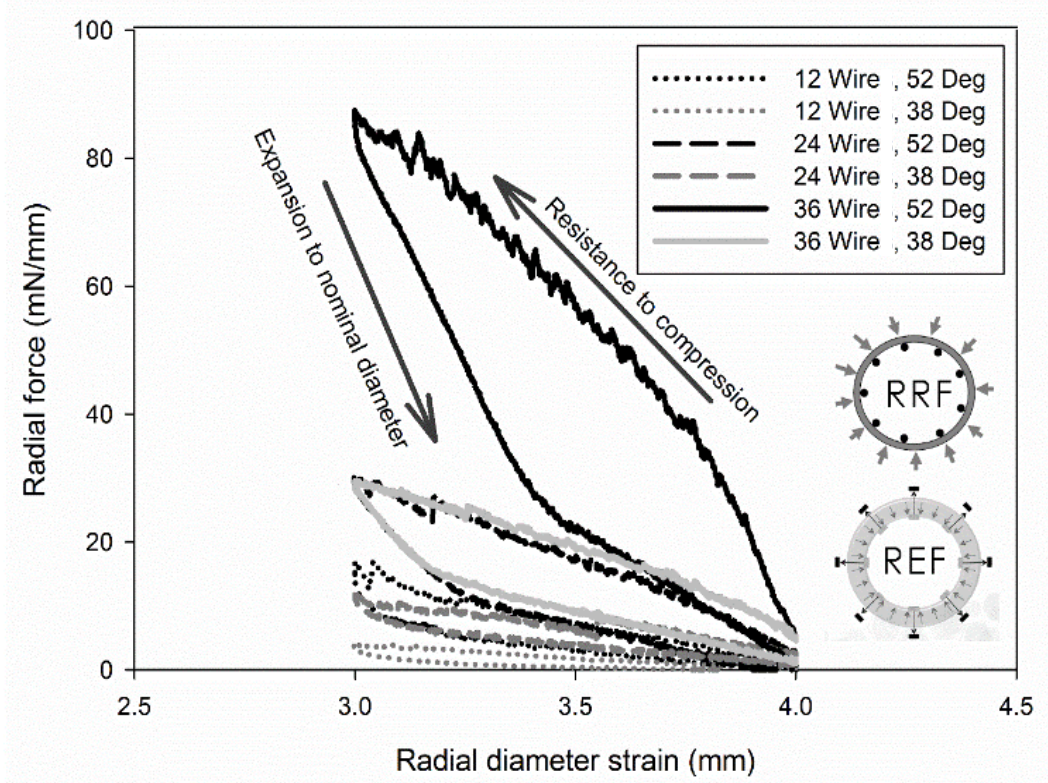

Fig. 5 Relationship between radial force and radial diameter of stents with various number of wires and braiding angle.

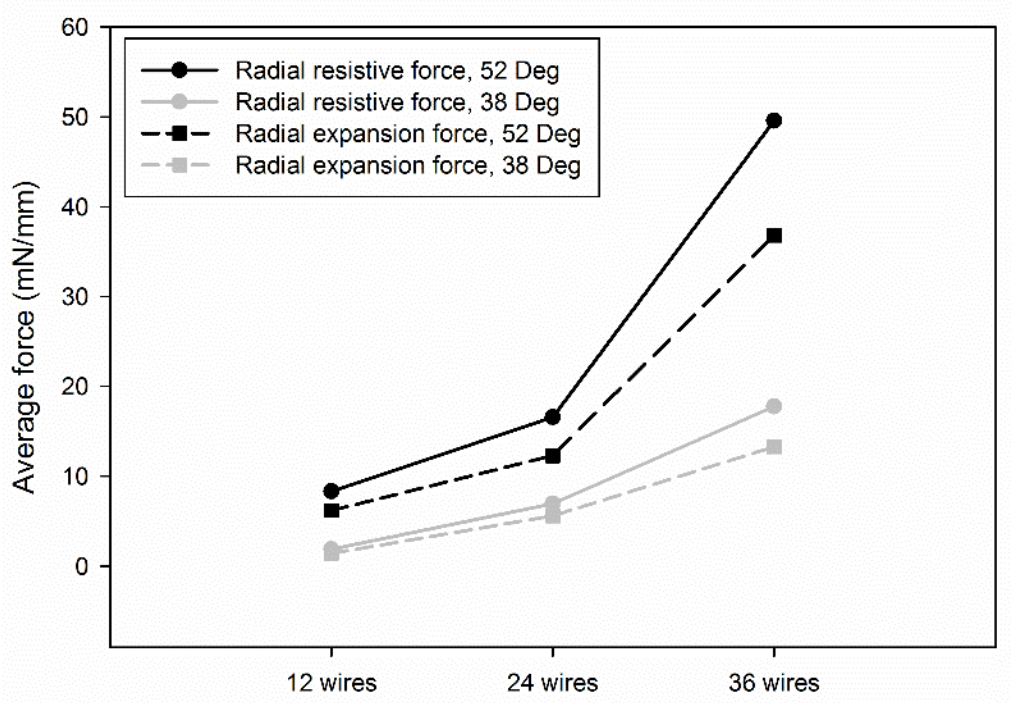

Fig. 6 Number of wire dependence of average radial force of stent with different braiding angle.

Fig. 7 shows the relationship between average radial force and bending force of stentriever with various number of wires and braiding angle. The 36 wire braided stent reveals the highest radial force and bending force. It is able to mention that this stent shows the outstanding properties for collectiong thrombus such as great radial resisitive force, high expanding force in radial direction. On the other hand, this stent still requires lower bending resistance in order to acheive stent with more flexibility. 


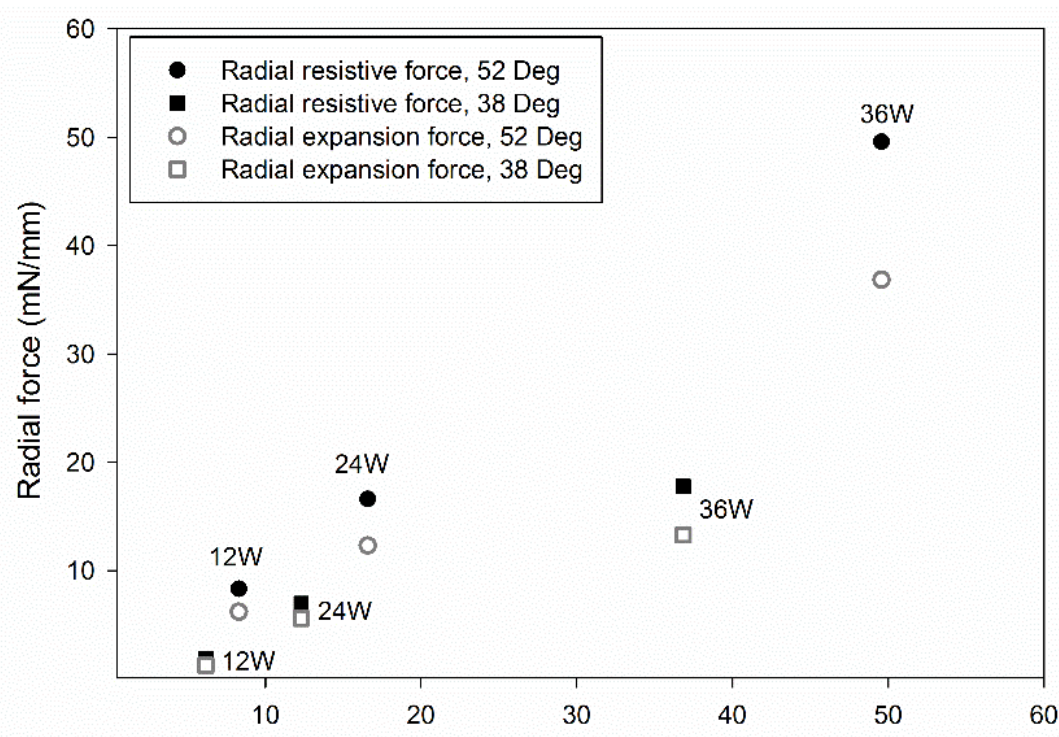

Fig. 7 Relationship between average radial force and bending force of stentriever with various number of wires and braiding angle.

\section{Conclusion}

In order to design and fabricate the braiding stentriever, two principal factors, Braiding angle and number of wires should be carefully determined to investigate their effects on mechanical properties. Increasing of braiding angle and number of wire results in increasing of radial resistive force, radial expansion force and three-point bending force. It is found that maximum radial resistive force, maximum radial expansion force and maximum bending force were confirmed at 50 $\mathrm{mN} / \mathrm{mm}, 37 \mathrm{mN} / \mathrm{mm}$ and $0.423 \mathrm{~N}$, respectively in 36 wire stent with a braiding angle of 52 degree. It is able to summarize that ideal stent needs to have high radial resistive force to maintain the stability from stent and low stiffness to bend during the delivery process in the blood vessel. In a future, more investigations and comparison with commercial stent and retrieved ability test will be required.

\section{Acknowledgement}

This work was supported by Research and Researcher for Industry (RRI) Fund, National research council Thailand (NRCT), and National research university project (NRU) and Smart Laboratory, King Mongkut's University of Technology Thonburi (KMUTT).

\section{References}

[1] World Stroke Day. [Online]. [Cited 2011 Aug 19]; Available from: URL: (http://www.worldstrokecampaign.org/media/Pages/AboutWorldStrokeDay2010.aspx)

[2] L. Thomassen, S. J. Bakke. Endovascular reperfusion therapy in acute ischemic stroke. Acta. Neurol. Scand. 187 (2007) 22-29.

[3] B. Katona, E. Bognar, B. Berta, et al. Chemical etching of Nitinol stent, Acta. Bioeng. Biomech. 15(4) (2013) 3-8.

[4] N. Muhammad, Laser micromachining of coronary stent for medical applications (Ph. D thesis), Retrieved from the University of Manchester., 2012. 